\title{
DIETA DE LITHOBATES ZWEIFELI HILLIS, FROST Y WEBB 1984 (ANURA: RANIDAE) EN UN RÍO ESTACIONAL DEL CENTRO DE MÉXICO
}

\author{
Luís Javier MENDOZA-ESTRADA, Rosmandi LARA LóPEZ \\ \& Rubén CASTRO-FranCO* \\ Laboratorio de Herpetología, Departamento de Biología Animal, Centro de Investigaciones \\ Biológicas, Universidad Autónoma del Estado de Morelos, Av. Universidad 1001, \\ Chamilpa 62209, Cuernavaca, Morelos, MÉXICO. (LJME) \\ luisjaviermendoza@yahoo.com.mx, (RLL) rlara@cib.uaem.mx, (RCF) \\ castro@cib.uaem.mx. \\ * Envío de correspondencia
}

\section{RESUMEN}

Por medio de lavado estomacal de 81 organismos se estudió la dieta de Lithobates zweifeli en un río estacional de Morelos, México. Los datos fueron analizados por épocas del año (lluvias y sequía), estadios de desarrollo y por sexos. Las presas fueron clasificadas por sus hábitos en terrestres o acuáticas, determinadas a nivel de orden, familia y género y algunas veces a especie. La dieta de $L$. zweifeli estuvo constituida por 102 componentes alimentarios, principalmente insectos de hábitos terrestres. Hemiptera (Cicadellidae), Lepidoptera (Noctuidae) y Megaloptera (Corydalus texanus) tuvieron los valores mas altos de ingestión y frecuencia en la dieta. Se encontraron 54 componentes alimentarios en la época de estiaje y 84 en la de lluvias. La amplitud del nicho trófico estimado a partir del método de Levin reveló valores bajos en ambas épocas del año. El alimento de las hembras estuvo constituido por 63 componentes, el de los machos por 55 y el de los juveniles por 49 . Una prueba de $J i$ cuadrada reveló que no existen diferencias significativas entre las dietas por estadio de desarrollo, sexos y épocas del año. El traslape de los nichos tróficos, estimado a partir del índice de MacArthur y Levin reveló valores bajos en todas las combinaciones por sexos y épocas del año. La vegetación flotante y riparia como hábitat de los insectos es importante para la alimentación de L. zweifeli.

Palabras clave: Hábitos, Alimentación, Lithobates zweifeli, Río estacional, México.

\begin{abstract}
The diet of Lithobates zweifeli was studied by means of stomach flushing in a seasonal river of Morelos, Mexico. Diet was analyzed by season (wet and dry), development stages and by sex. Food items were classified by their habits in terrestrial or aquatic prey, and determined to order, family, genus and sometimes to species. The diet of L. zweifeli comprised 102 nutritional components, mainly land habits insects. Hemiptera (Cicadellidae), Lepidoptera (Noctuidae) and Megaloptera (Corydalus texanus) had the highest values of ingestion and frecuence in the diet. We found 54 nutritional components in the dry season, and 84 in the rainy season. The estimated amplitude of trophic niche by the Levin's method revealed low values in both seasons. Diet was constituted by 63 nutritional
\end{abstract}


components in females, 55 in males and 49 in juveniles. A Chi-squared test showed not significant differences in the diet by development stage, sex, and season. The overlap of the trophic niches estimated by the MacArthur and Levin index revealed low value in sex and season combinations. The floating and riparian vegetation as insects' habit is important for the feeding of L. zweifeli.

Key words: Habits, feeding, Lithobates zweifeli, seasonal river, Mexico.

\section{INTRODUCCIÓN}

El estudio de los hábitos de alimentación en anfibios anuros es importante por el papel que desempeñan estos organismos en los ecosistemas acuáticos y terrestres (Hirai \& Matsui 1999). Estudios previos sobre la dieta de este grupo (Hayes \& Tennant 1985, Werner et. al. 1995, Menéndez-Guerrero 2001) indican que muchas especies en su fase adulta son carnívoras debido a que se alimentan principalmente de insectos (Duellman \& Trueb 1994) y en algunos casos llegan a consumir otros anuros (Cogalniceanu et. al. 2001), pequeños mamíferos y aves, e incluso materia vegetal (Daza - Vaca \& Castro Herrera 1999). El consumo de materia vegetal es atribuido a una ingestión accidental durante la captura de presas y no como complemento en la dieta en los anuros adultos (Evans \&Lampo 1996, Anderson et al. 1999); sin embargo, se sabe que ocurre en salamandras, en sapos como Chaunus marinus (Duellman \& Trueb 1994, CloudsleyThompson 1999), en la rana Hyla truncata que consume frutos como parte de su dieta (da Silva et al. 1989, da Silva \& Britto-Pereira 2006) y en Zachaenus parvulus (Van Sluys et al. 2001). Lithobates zweifeli es una especie de rana endémica de México, habitante de cuerpos de agua tropicales en áreas de selva baja caducifolia (CastroFranco et. al. 2006) y su dieta es aun desconocida. En México se han realizado pocos estudios sobre la dieta de ranas (Rodríguez-Blanco 1990, Ramírez-Bautista \& LemosEspinal 2004). En este trabajo se presenta información sobre el uso de recursos alimentarios en una población de Lithobates zweifeli que vive en un río tropical en la región centro sur de Morelos, México.

\section{MATERIALES Y METODOS}

\section{Área de estudio}

Se localiza $5 \mathrm{~km}$ al sur de Yautepec, Morelos, México (18 49' 20" Latitud N, $99^{\circ}$ 05' 37' Longitud O, altitud 1,105 msnm), en aproximadamente $1 \mathrm{~km}$ de extensión a lo largo del Río Yautepec. El clima es del tipo cálido subhúmedo [Awo"(i)g] con lluvias en verano y un porcentaje de lluvia invernal menor de cinco por ciento, la precipitación media anual oscila entre 800 y $1,000 \mathrm{~mm}$, temperatura media anual superior a $22^{\circ} \mathrm{C}$, cociente $\mathrm{P} / \mathrm{T}$ menor de 43.2 , la máxima precipitación ocurre en 
septiembre con $200 \mathrm{~mm}$, la mínima entre diciembre y marzo con menos de $5.0 \mathrm{~mm}$. Hay una canícula a mitad del verano, la temperatura más alta $\left(26^{\circ} \mathrm{C}\right.$ a $\left.27^{\circ} \mathrm{C}\right)$ se presenta en mayo y la más baja $\left(20^{\circ} \mathrm{C}\right.$ a $21^{\circ} \mathrm{C}$ ) entre enero y diciembre (García 1988). En esta localidad hay un periodo de estiaje que dura siete meses, de noviembre a mayo, y un periodo de lluvias de junio a octubre. En el estiaje el río tiene poca corriente y permite la formación de_pozas aisladas con agua estancada, en lluvias la corriente es continua con poca velocidad y estas condiciones dan al río su carácter estacional.

\section{Obtención de los datos}

Las capturas fueron realizadas dos días de cada mes entre el 5 de octubre de 2001 y el 3 de octubre de 2002, entre las 20:00 y las 23:00 horas. Por observaciones previas, se determinó este horario como el de mayor probabilidad para encontrar a las ranas con los estómagos llenos de alimento, aun sin digerir. La búsqueda de las ranas se hizo con lámparas en ambas orillas del río, remansos, huecos de troncos, raíces y bajo piedras. De cada ejemplar capturado obtuvimos la longitud hocicocloaca (LHC) con precisión de $0.1 \mathrm{~mm}$ y el peso con precisión de $0.1 \mathrm{~g}$. El sexo se determinó mediante el análisis de caracteres morfológicos (Hillis et. al. 1984) y la observación de gónadas en algunos ejemplares que fueron preservados. Como juveniles fueron considerados individuos transformados de tamaño pequeño (40.0$70.0 \mathrm{~mm}$ LHC). El contenido de los estómagos fue extraído por el método de lavado estomacal (Legler \& Sullivan 1979), tratando de que fuera lo más pronto posible a partir del momento de la captura. Las muestras de contenido estomacal fueron conservadas en alcohol al $70 \%$ y las ranas fueron liberadas en el lugar donde fueron capturadas. Los diversos grupos de alimentos fueron determinados al nivel taxonómico de familia, y en algunos casos fue posible hasta género y especie. El reconocimiento de las categorías de alimento se hizo en un microscopio estereoscópico Nikon modelo SMZ-2B y se consultó literatura adecuada para cada grupo. Las presas determinadas fueron clasificadas en presas de hábitos acuáticos y presas de hábitos terrestres. Las presas que se pueden encontrar tanto en ambientes acuáticos como terrestres no fueron consideradas.

\section{Análisis de la información}

Para cuantificar los contenidos estomacales se calculó el porcentaje de ingestión (PI) y la frecuencia de ocurrencia (FO). El primero fue estimado a través del método numérico por cuadrícula (Windell \& Bowen 1978), el cual consiste en el uso de una cuadrícula milimétrica de 5 x $5 \mathrm{~cm}$ colocada en el fondo externo de una caja Petri, el contenido estomacal se extiende homogéneamente y el número total de cuadros que ocupan los distintos tipos de alimento de un mismo estómago representa el $100 \%$ en la dieta de ese individuo; el porcentaje de cada grupo ingerido es el número de 
cuadros dividido entre el total y multiplicado por 100. La frecuencia de ocurrencia es el número de veces en que aparecen los diferentes tipos de alimento en el contenido estomacal (Lagler 1977), por lo tanto los resultados no se ajustan al 100\%. La FO de cada componente alimentario al ser dividido entre $100 \mathrm{~m}$ se conoce como índice de Albertaine (1973) (IA), y se compara con una escala en la cual valores de 0 a 0.10 ubican al componente alimentario como ingerido en forma accidental, valores de 0.11 a 0.50 indican una ingestión ocasional o secundaria y valores de 0.51 a 1.0 indican alimentos ingeridos en forma preferente. Para evaluar la amplitud del nicho trófico se utilizó el índice de Levin (1968) (IL) en una escala de 0 a 1, donde todos los valores cercanos a cero indican que los depredadores ingieren pocos componentes alimentarios, es decir, tienen mínima amplitud de nicho trófico y en consecuencia máxima especialización; valores cercanos a 1 denotan nichos tróficos amplios (Hurlbert 1978). Para medir el traslape de nicho se utilizó el índice de MacArthur \& Levins (1967):

$$
M_{j k}=\frac{\sum_{i}^{n} p^{i j} p_{i k}}{\sum p^{2} i j}
$$

donde $M_{j k}$ es el traslape de nicho de la especie $k$ sobre la especie $j ; p_{i j}$ es la proporción del recurso $i$ del total de recursos usados entre los sexos, adultos y jóvenes $j, p_{i k}$ es la proporción del recurso $i$ del total de recursos usados por la especie $k$, y $n$ es el número de los recursos. Un valor de cero indica que los recursos no tienen uso común, y un valor cercano a uno indica traslape máximo en el uso de recursos. Para determinar diferencias significativas en los componentes de la dieta por sexos y épocas del año, se aplicó una prueba de $J i$-cuadrada (Siegel 1976).

\section{RESULTADOS}

Dieta general. Obtuvimos contenidos estomacales de 81 especimenes de Lithobates zweifeli de los cuales 25 fueron machos $(\mathrm{LHC}=75.3 \pm 4.12 \mathrm{~mm}$; peso= $62 \pm 13.6 \mathrm{~g}), 27 \mathrm{hembras}(\mathrm{LHC}=90.7 \pm 4.3 \mathrm{~mm}$; peso $=100.4 \pm 18.4 \mathrm{~g})$ y 29 juveniles $($ LHC $54.6 \pm 13.7 \mathrm{~mm}$; peso $=25.0 \pm 14.7 \mathrm{~g})$. El $88.8 \%$ de los estómagos $(n=72)$ contuvieron al menos un componente alimentario y el $11.2 \%(n=9)$ estuvieron vacíos, por lo que no fueron considerados en los análisis. La dieta de las ranas estuvo constituida por 102 componentes de origen animal que pertenecen a ocho clases, 21 órdenes, 76 familias, 46 géneros y ocho especies (Anexo 1). La mayoría fueron invertebrados y en menor proporción algunos vertebrados como peces de la familia Cichlidae y larvas de ranas de Lithobates sp., probablemente conespecíficos. Los componentes alimentarios que no pudieron ser determinados debido al avanzado estado de digestión fueron clasificados como restos animales indeterminados. Se 
encontró además materia mineral (arena y piedra pequeñas) en el $6.9 \%$, y materia vegetal (corteza, flores, tallos, hojas, foliolos y pastos) en el $29.1 \%$ del total de estómagos analizados.

Variación estacional de la dieta. Hubo diferencias en la dieta en los períodos de estiaje y de lluvias. En los 32 estómagos de las ranas capturadas (15 hembras, 9 juveniles y 11 machos) en época de estiaje se registraron 54 componentes alimentarios (Cuadro 1). Insectos de las familias Noctuidae (Lepidoptera), Odonata y Opilionida del género Leiobonum sp., mostraron los PI más altos con $10.1 \%, 6.4$ $\%$ y $6.8 \%$ respectivamente; sin embargo, por sus FO con $30.0 \%, 5.0 \%$ y $30.0 \%$ respectivamente, fueron considerados como alimentos secundarios de acuerdo con el IA calculado. Renacuajos de Lithobates sp. tuvieron un PI de $18.2 \%$; sin embargo, de acuerdo con el valor del IA (0.15) fueron considerados como alimento secundario, al igual que los restos de animales indeterminados $(\mathrm{IA}=0.25)$. En los estómagos de individuos capturados en la época de estiaje se encontraron piedras $(10 \%)$ y materia vegetal (45\%). Las presas de hábitos terrestres representaron el $78.7 \%$ de las presas consumidas en la época de estiaje y las de hábitos acuáticos representaron el $21.3 \%$. El valor relativamente bajo de IL (0.22) obtenido para la amplitud del nicho trófico, revela que en esta época del año las ranas ingieren pocos componentes alimentarios.

En la época de lluvias se analizaron estómagos de 49 individuos ( 15 hembras, 20 juveniles y 14 machos) en los que se encontraron 79 componentes alimentarios de origen animal (Cuadro 2). Lepidópteros de la familia Noctuidae, Lepidoptera indeterminados, Corydalus texanus (Megaloptera), y dípteros del género Simulium sp. tuvieron los valores de PI más altos con $17.7 \%, 12.1 \%, 24.2 \%$ y $8.9 \%$ respectivamente. Los lepidópteros de la familia Noctuidae con una FO del 61.5\%, fueron considerados como alimento preferente durante esta época del año de acuerdo con el IA $=0.61$, no así los demás grupos que fueron considerados como alimentos secundarios con FO de $34.6 \%$, $21.1 \%$ y $25 \%$ en el mismo orden. Peces como el cíclido Cichlasoma nigrofasciatum y renacuajos de Lithobates sp., mostraron valores bajos de PI y FO, por lo que fueron considerados como alimentos de consumo accidental. Los restos de animales no determinados tuvieron un PI bajo con $2.7 \%$, y de acuerdo con el valor de FO de $26.9 \%$ fueron considerados como alimento secundario. Se encontró materia de origen mineral (piedras y arena) en el $5.7 \%$ y materia vegetal en el $23.0 \%$ de los estómagos analizados en este periodo. Las presas de hábitos terrestres representaron el $75.3 \%$ de las presas consumidas durante la época de lluvias y las de hábitos acuáticos representaron el $24.7 \%$. El valor de IL (0.12) para la amplitud de nicho trófico es menor casi en $50 \%$ que el observado en la época de estiaje.

Dieta de juveniles. Se analizaron 29 estómagos, de los cuales 24 contenían alimento. La dieta estuvo constituida por 49 componentes alimentarios (Cuadro 3), todos invertebrados. Lepidópteros de la familia Noctuidae, lepidópteros no 
determinados y dípteros del género Simulium sp., tuvieron los PI más altos con el $34.2 \%, 24.8 \%$ y $9.0 \%$ respectivamente. Por su FO los Noctuidae con $83.3 \%$ y el valor obtenido del IA fueron considerados como alimento preferente, y los otros dos como secundarios $45.8 \%$ y $20.8 \%$ en el mismo orden. Se encontró materia mineral (piedras) en el $12.5 \%$, y materia vegetal en el $20.8 \%$ de los estómagos analizados. En la dieta de los juveniles, las presas de hábitos terrestres representaron un $77.8 \%$, y las presas acuáticas el $22.2 \%$. La amplitud de nicho trófico tuvo un valor bajo (IL= $0.12)$.

Dieta de las hembras. Se analizaron 27 estómagos de los cuales sólo cuatro no contuvieron alimento. La dieta estuvo constituida por 63 componentes alimentarios incluyendo invertebrados y vertebrados (Cuadro 4). Megalópteros como Corydalus texanus, tuvieron el PI más alto con $39.3 \%$, seguido por anélidos oligoquetos con 6.3 $\%$; sin embargo, ambos tipos de presas fueron considerados como alimentos secundarios por sus valores de IA y FO con $16.6 \%$ y $41.6 \%$ respectivamente. El resto de los componentes del grupo de invertebrados tuvieron porcentajes de ingestión y preferencia bajos, por lo que fueron considerados como alimentos de ingestión accidental. Los vertebrados estuvieron representados por estadios larvarios de Lithobates sp., que tuvieron un PI y FO relativamente bajos con $3.1 \%$ y $7.1 \%$ respectivamente, y de acuerdo con el valor de IA (0.07) fueron considerados como alimento accidental, al igual que los restos de animales no determinados con el 7.1 $\%$ de FO. En el $41.6 \%$ de los estómagos analizados de las hembras se encontró materia vegetal y en el $4.1 \%$ materia mineral (piedras). Las presas de hábitos terrestres tuvieron un porcentaje de $77.6 \%$ y las presas acuáticas un porcentaje de $22.4 \%$. La amplitud del nicho trófico de las hembras ( $\mathrm{IL}=0.09)$ fue el más bajo.

Dieta de los machos. Se analizaron 25 estómagos y todos presentaron al menos un componente alimentario (Cuadro 5). La dieta estuvo constituida por 55 componentes alimentarios de origen animal. Lepidópteros de la familia Noctuidae presentaron el PI más alto con $19.3 \%$; sin embargo, fueron considerados como alimento secundario por su IA y FO con $45.8 \%$. Los megalópteros Corydalus texanus, mostraron un PI de $5.9 \%$, y por su valor de IA bajo con 0.08 , fueron considerados como alimento de consumo accidental. El resto de los componentes alimentarios del grupo de invertebrados, tuvieron porcentajes de ingestión y preferencia bajos, por lo que fueron considerados como alimentos de consumo accidental. Los vertebrados estuvieron representados por larvas de Lithobates sp., que tuvieron un PI relativamente alto de $13.7 \%$, sin embargo, debido al valor de IA de 0.08 , fueron considerados como alimento de consumo accidental. Los restos de animales no determinados, por su IA y FO fueron considerados como alimento secundario con $29.2 \%$. La materia vegetal estuvo presente en el $25 \%$ y la materia mineral (piedras) únicamente en el $4.17 \%$. Del total de presas en la dieta de los machos, el $71.4 \%$ son presas de hábitos terrestres y el $28.6 \%$ presas de hábitos 
acuáticos. La amplitud de nicho trófico tuvo un valor bajo $(\mathrm{IL}=0.26)$, pero en comparación con las hembras y juveniles fue el más alto de los tres. No existen diferencias significativas entre las dietas por sexos (hembra-machos $\chi^{2}=0.09, P \leq$ 0.05 ) por estadios de desarrollo (hembras-juveniles $\chi^{2}=0.09, P \leq 0.05$; machosjuveniles $\left.\chi^{2}=1.6, P \leq 0.05\right)$ ni por épocas del año $\left(\chi^{2}=0.04, P \leq 0.05\right)$.

Traslape de nicho trófico. No se registraron traslapes significativos de los nichos de Lithobates zweifeli por épocas del año $\left(M_{j k}=0.29\right)$ en las combinaciones entre los sexos, entre los juveniles y hembras $\left(M_{j k}=0.12\right)$, ni entre los juveniles $v s$. machos y hembras vs. machos $\left(M_{j k}=0.40\right)$, asumiendo que un valor $\geq 0.60$ es significativo (Zaret \& Rand 1971).

\section{Cuadro 1}

Composición de la dieta de Lithobates zweifeli en época de estiaje, de acuerdo con el porcentaje de ingestión (PI), frecuencia de ocurrencia (FO) e índice de Albertaine (IA). Los hábitos de las presas son: $\mathrm{A}=$ Acuático, $\mathrm{T}=$ Terrestre, $\mathbb{K}=$ Ambiguo $\mathrm{y} æ=$ Desconocido.

\begin{tabular}{|c|c|c|c|c|}
\hline Componentes alimentarios & PI (\%) & FO $(\%)$ & IA & Hábito \\
\hline Materia vegetal & - & 45.0 & - & \\
\hline \multicolumn{5}{|l|}{ Moluscos Gasterópodos } \\
\hline \multicolumn{5}{|l|}{ Planorbidae } \\
\hline Helisoma $\mathrm{sp}$. & 3.70 & 5.0 & 0.05 & A \\
\hline \multicolumn{5}{|l|}{ Isópodos } \\
\hline Oniscidae & 3.45 & 15.0 & 0.15 & $\mathrm{~T}$ \\
\hline \multicolumn{5}{|l|}{ Arácnidos } \\
\hline Amaurobiidae & 0.09 & 5.0 & 0.05 & $\mathrm{~T}$ \\
\hline \multicolumn{5}{|l|}{ Buthidae } \\
\hline Centruroides l. limpidus & 4.70 & 15.0 & 0.15 & $\mathrm{~T}$ \\
\hline Dipluridae & 0.15 & 5.0 & 0.05 & $\mathrm{~T}$ \\
\hline Dysderidae & 0.14 & 5.0 & 0.05 & $\mathrm{~T}$ \\
\hline Lycosidae & 4.18 & 20.0 & 0.20 & $\mathrm{~T}$ \\
\hline Theridiidae & 0.23 & 15.0 & 0.15 & $\mathrm{~T}$ \\
\hline Uloboridae & 0.27 & 5.0 & 0.05 & $\mathrm{~T}$ \\
\hline Indeterminados & 0.14 & 10.0 & 0.10 & $\mathrm{~T}$ \\
\hline \multicolumn{5}{|l|}{ Opiliones } \\
\hline \multicolumn{5}{|l|}{ Sclerosomatidae } \\
\hline Leiobonum sp. & 6.88 & 30.0 & 0.30 & $\mathrm{~T}$ \\
\hline
\end{tabular}


Continúa Cuadro 1

\begin{tabular}{|c|c|c|c|c|}
\hline Componentes alimentarios & PI (\%) & FO $(\%)$ & IA & Hábito \\
\hline \multicolumn{5}{|l|}{ Insectos } \\
\hline \multicolumn{5}{|l|}{ Coleópteros } \\
\hline \multicolumn{5}{|l|}{ Curculionidae } \\
\hline Auleutes sp. & 0.20 & 5.0 & 0.05 & A \\
\hline Elateridae & 0.11 & 5.0 & 0.05 & $\mathrm{~T}$ \\
\hline Lampiridae & 0.05 & 5.0 & 0.05 & $\mathrm{~T}$ \\
\hline Staphilinidae & 0.03 & 5.0 & 0.05 & $\mathrm{~T}$ \\
\hline Tenebrionidae & 0.44 & 5.0 & 0.05 & $\mathrm{~T}$ \\
\hline Indeterminados & 0.11 & 5.0 & 0.05 & $æ$ \\
\hline \multicolumn{5}{|l|}{ Dermapteros } \\
\hline \multicolumn{5}{|l|}{ Forficulidae } \\
\hline Doru lineare & 1.40 & 15.0 & 0.15 & $\mathrm{~T}$ \\
\hline \multicolumn{5}{|l|}{ Dípteros } \\
\hline \multicolumn{5}{|l|}{ Ceratopogonidae } \\
\hline Dasyhelea saltensis & 2.34 & 5.0 & 0.05 & $\mathrm{~T}$ \\
\hline \multicolumn{5}{|l|}{ Culicidae } \\
\hline Culex sp. & 0.62 & 5.0 & 0.05 & $\mathrm{~T}$ \\
\hline \multicolumn{5}{|l|}{ Dolichopodidae } \\
\hline \multicolumn{5}{|l|}{ Drosophilidae } \\
\hline Drosophila sp. & 0.08 & 5.0 & 0.05 & $\mathrm{~T}$ \\
\hline \multicolumn{5}{|l|}{ Stratiomyidae } \\
\hline Stratiomys sp. & 0.14 & 10.0 & 0.10 & $\mathrm{~T}$ \\
\hline \multicolumn{5}{|l|}{ Tipulidae } \\
\hline \multirow{2}{*}{\multicolumn{5}{|c|}{ Efemerópteros }} \\
\hline & & & & \\
\hline \multicolumn{5}{|l|}{ Leptohyphidae } \\
\hline Tricorythodes sp. (larvas) & 0.03 & 5.0 & 0.05 & $\mathrm{~A}$ \\
\hline \multicolumn{5}{|l|}{ Hemípteros } \\
\hline \multicolumn{5}{|l|}{ Anthocoridae } \\
\hline Anthocoris sp. & 0.11 & 5.0 & 0.05 & $\mathrm{~T}$ \\
\hline Corixidae & 2.17 & 5.0 & 0.05 & $\mathrm{~T}$ \\
\hline \multicolumn{5}{|l|}{ Gelastocoridae } \\
\hline Nerthra sp. & 4.78 & 20.0 & 0.20 & A \\
\hline Lygaeidae & 4.37 & 25.0 & 0.25 & $\mathrm{~T}$ \\
\hline \multicolumn{5}{|l|}{ Nepidae } \\
\hline Ranatra sp. & 0.39 & 5.0 & 0.05 & A \\
\hline Reduviidae & 0.59 & 15.0 & 0.15 & $\mathrm{~T}$ \\
\hline Indeterminados & 0.16 & 10.0 & 0.10 & $æ$ \\
\hline \multicolumn{5}{|l|}{ Himenópteros } \\
\hline Adrenidae & 0.33 & 10.0 & 0.10 & $\mathrm{~T}$ \\
\hline Chalcididae & 0.19 & 5.0 & 0.05 & $\mathrm{~T}$ \\
\hline
\end{tabular}

176 
Concluye Cuadro 1

\begin{tabular}{|c|c|c|c|c|}
\hline Componentes alimentarios & PI (\%) & FO (\%) & $\mathbf{I A}$ & Hábito \\
\hline \multicolumn{5}{|l|}{ Formicidae } \\
\hline Atta mexicana & 0.42 & 5.0 & 0.05 & $\mathrm{~T}$ \\
\hline Camponotus atriceps & 1.45 & 20.0 & 0.20 & $\mathrm{~T}$ \\
\hline Indeterminados & 0.02 & 5.0 & 0.05 & $\mathrm{~T}$ \\
\hline \multicolumn{5}{|l|}{ Homópteros } \\
\hline Cicadellidae & 0.19 & 5.0 & 0.05 & $\mathrm{~T}$ \\
\hline Typhlocybinae & 0.30 & 5.0 & 0.05 & $\mathrm{~T}$ \\
\hline Cicadidae & 1.86 & 5.0 & 0.05 & $\mathrm{~T}$ \\
\hline \multicolumn{5}{|l|}{ Lepidopteros } \\
\hline Noctuidae & 10.19 & 30.0 & 0.30 & $\mathrm{~T}$ \\
\hline Indeterminados & 0.30 & 10.0 & 0.10 & $æ$ \\
\hline \multicolumn{5}{|l|}{ Megalopteros } \\
\hline \multicolumn{5}{|l|}{ Corydalidae } \\
\hline Corydalus texanus & 4.23 & 5.0 & 0.05 & Ж \\
\hline \multicolumn{5}{|l|}{ Odonatos } \\
\hline Aehsnidae (larvas) & 4.00 & 5.0 & 0.05 & A \\
\hline Libellulidae & 0.55 & 5.0 & 0.05 & $\mathrm{~T}$ \\
\hline Indeterminados & 6.40 & 5.0 & 0.05 & Ж \\
\hline \multicolumn{5}{|l|}{ Orthopteros } \\
\hline Gryllidae & 3.78 & 15.0 & 0.15 & $\mathrm{~T}$ \\
\hline Indeterminados & 0.52 & 5.0 & 0.05 & $\mathrm{~T}$ \\
\hline \multicolumn{5}{|l|}{ Trichopteros } \\
\hline \multicolumn{5}{|l|}{ Hydropsichidae } \\
\hline Leptonema sp. & 0.01 & 5.0 & 0.05 & $\mathrm{~T}$ \\
\hline Hydroptilidae & 0.34 & 5.0 & 0.05 & A \\
\hline \multicolumn{5}{|l|}{ Odontoceridae } \\
\hline Marilia $\mathrm{sp}$ & 0.39 & 5.0 & 0.05 & $\mathrm{~T}$ \\
\hline \multicolumn{5}{|l|}{ Peces } \\
\hline Cichlidae & 0.27 & 5.0 & 0.05 & A \\
\hline \multicolumn{5}{|l|}{ Anfibios anuros } \\
\hline \multicolumn{5}{|l|}{ Ranidae } \\
\hline Lithobates sp. (larvas) & 18.26 & 15.0 & 0.15 & A \\
\hline Materia animal mixta indeterminada & 3.45 & 25.0 & 0.25 & $æ$ \\
\hline Materia mineral (piedras) & - & 10.0 & - & \\
\hline
\end{tabular}


Mendoza Estrada et al.: Dieta de Lithobates sweifeli Hillis, Frost y Webb 1984 (Anura: Ranidae)

\section{Cuadro 2}

Composición de la dieta de Lithobates zweifeli en la época de lluvias, de acuerdo con el porcentaje de ingestión (PI), frecuencia de ocurrencia (FO) e índice Albertaine (IA). Los hábitos de las presas son: $\mathrm{A}=$ Acuático, $\mathrm{T}=$ Terrestre, $\mathrm{K}_{=}$Ambiguo y $æ=$ Desconocido.

\begin{tabular}{|c|c|c|c|c|}
\hline Componentes alimentarios & PI (\%) & FO (\%) & IA & Hábito \\
\hline Materia vegetal & - & 23.00 & - & \\
\hline \multicolumn{5}{|l|}{ Anélidos } \\
\hline Oligoqueta & 3.82 & 7.69 & 0.07 & $\mathrm{~T}$ \\
\hline \multicolumn{5}{|l|}{ Moluscos Gasterópodos } \\
\hline \multicolumn{5}{|l|}{ Lymnaeidae } \\
\hline Physa sp. & 0.10 & 3.85 & 0.03 & A \\
\hline Fossaria sp. & 0.09 & 1.92 & 0.01 & A \\
\hline Quilópodos Geophilomorpha & 0.83 & 3.85 & 0.03 & $\mathrm{~T}$ \\
\hline \multicolumn{5}{|l|}{ Arácnidos } \\
\hline Dipluridae & 0.02 & 1.92 & 0.01 & $\mathrm{~T}$ \\
\hline Lycosidae & 4.25 & 25.0 & 0.25 & $\mathrm{~T}$ \\
\hline Pholcidae & 0.01 & 1.92 & 0.01 & $\mathrm{~T}$ \\
\hline Salticidae & 0.02 & 1.92 & 0.01 & $\mathrm{~T}$ \\
\hline Tetragnathidae & 0.18 & 1.92 & 0.01 & $\mathrm{~T}$ \\
\hline Zodariidae & 0.19 & 1.92 & 0.01 & $\mathrm{~T}$ \\
\hline \multicolumn{5}{|l|}{ Opiliones } \\
\hline \multicolumn{5}{|l|}{ Sclerosomatidae } \\
\hline Leiobonum sp. & 0.37 & 7.69 & 0.07 & $\mathrm{~T}$ \\
\hline \multicolumn{5}{|l|}{ Insectos } \\
\hline Coleópteros & 0.34 & 5.77 & 0.05 & $\mathrm{~T}$ \\
\hline \multicolumn{5}{|l|}{ Carabidae } \\
\hline Selenophores sp. & 0.11 & 1.92 & 0.01 & $\mathrm{~T}$ \\
\hline Stenomorphus sp. & 0.22 & 1.92 & 0.01 & $\mathrm{~T}$ \\
\hline Indeterminados & 1.24 & 15.38 & 0.15 & $\mathrm{~T}$ \\
\hline \multicolumn{5}{|l|}{ Cicindelidae } \\
\hline Cicindella sp. & 1.39 & 11.54 & 0.115 & $\mathrm{~T}$ \\
\hline Curculionidae & 0.18 & 3.85 & 0.039 & $\mathrm{~T}$ \\
\hline Rodobaenus sp. & 0.08 & 1.92 & 0.019 & $\mathrm{~T}$ \\
\hline Elateridae & 0.35 & 5.77 & 0.058 & $\mathrm{~T}$ \\
\hline \multicolumn{5}{|l|}{ Hydrophilidae } \\
\hline Hydrophilus sp. (larvas) & 1.75 & 17.31 & 0.173 & A \\
\hline Lampiridae & 0.08 & 3.85 & 0.039 & $\mathrm{~T}$ \\
\hline \multicolumn{5}{|l|}{ Melolonthidae } \\
\hline Cyclocephala sp. & 0.98 & 3.85 & 0.039 & $\mathrm{~T}$ \\
\hline Diplotaxis sp. & 0.07 & 1.92 & 0.019 & $\mathrm{~T}$ \\
\hline
\end{tabular}


Continúa Cuadro 2

\begin{tabular}{|c|c|c|c|c|}
\hline Componentes alimentarios & PI (\%) & FO $(\%)$ & IA & Hábito \\
\hline \multicolumn{5}{|l|}{ Scarabeidae } \\
\hline Aphodius sp. & 0.03 & 3.85 & 0.039 & $\mathrm{~T}$ \\
\hline Staphilinidae & 0.02 & 1.92 & 0.019 & $\mathrm{~T}$ \\
\hline Tenebrionidae & 0.12 & 1.92 & 0.019 & $\mathrm{~T}$ \\
\hline \multicolumn{5}{|l|}{ Colémbolos } \\
\hline \multicolumn{5}{|l|}{ Sminthurididae } \\
\hline Sminthurides sp. & 0.02 & 3.85 & 0.039 & $\mathrm{~A}$ \\
\hline \multicolumn{5}{|l|}{ Dermapteros } \\
\hline \multicolumn{5}{|l|}{ Forficulidae } \\
\hline Doru lineare & 0.93 & 5.77 & 0.058 & $\mathrm{~T}$ \\
\hline \multicolumn{5}{|l|}{ Dípteros } \\
\hline \multicolumn{5}{|l|}{ Ceratopogonidae } \\
\hline Dasyhelea saltensis & 0.01 & 1.92 & 0.019 & $\mathrm{~T}$ \\
\hline Chironomidae (larvas) & 0.27 & 3.85 & & A \\
\hline \multicolumn{5}{|l|}{ Dolichopodidae } \\
\hline Dolichopus sp. & 0.03 & 1.92 & 0.019 & $\mathrm{~T}$ \\
\hline \multicolumn{5}{|l|}{ Drosophilidae } \\
\hline \multicolumn{5}{|l|}{ Lycoridae } \\
\hline Sciara $\mathrm{sp}$ & 0.12 & 7.69 & 0.077 & $\mathrm{~T}$ \\
\hline \multicolumn{5}{|l|}{ Simuliideae } \\
\hline Simulium sp. & 8.97 & 25.0 & 0.250 & A \\
\hline \multicolumn{5}{|l|}{ Stratiomyidae } \\
\hline Stratiomys sp. & 0.17 & 1.92 & 0.019 & $\mathrm{~T}$ \\
\hline \multicolumn{5}{|l|}{ Tipulidae } \\
\hline Tipula sp. & 0.60 & 1.92 & 0.019 & A \\
\hline \multicolumn{5}{|l|}{ Efemerópteros } \\
\hline \multicolumn{5}{|l|}{ Baetidae } \\
\hline Baetodes sp. (larvas) & 0.02 & 3.85 & 0.039 & A \\
\hline Camelobaetidius sp. (larvas) & 0.03 & 1.92 & 0.019 & A \\
\hline \multicolumn{5}{|l|}{ Leptohyphidae } \\
\hline Leptohyphes sp. (larvas) & 0.01 & 1.92 & 0.019 & A \\
\hline \multicolumn{5}{|l|}{ Hemípteros } \\
\hline \multicolumn{5}{|l|}{ Aetalionidae } \\
\hline Aetalion sp. & 0.22 & 9.62 & 0.096 & $\mathrm{~T}$ \\
\hline Coreidae & 1.21 & 5.77 & 0.058 & $\mathrm{~T}$ \\
\hline Lygaeidae & 0.47 & 13.46 & 0.135 & $\mathrm{~T}$ \\
\hline Miridae & 0.01 & 1.92 & 0.019 & $\mathrm{~T}$ \\
\hline Nabidae & 0.03 & 1.92 & 0.019 & $\mathrm{~T}$ \\
\hline \multicolumn{5}{|l|}{ Nepidae } \\
\hline Ranatra sp. & 0.15 & 1.92 & 0.019 & A \\
\hline
\end{tabular}


Continúa Cuadro 2

\begin{tabular}{|c|c|c|c|c|}
\hline Componentes alimentarios & PI (\%) & FO (\%) & IA & Hábito \\
\hline Pentatomidae & 0.05 & 1.92 & 0.019 & $\mathrm{~T}$ \\
\hline \multicolumn{5}{|l|}{ Veliidae } \\
\hline Microvelia sp. & 0.02 & 1.92 & 0.019 & A \\
\hline Rhagovellia sp. & 0.01 & 1.92 & 0.019 & $\mathrm{~T}$ \\
\hline Indeterminados & 0.71 & 3.85 & 0.039 & $æ$ \\
\hline \multicolumn{5}{|l|}{ Himenópteros } \\
\hline Figitidae & 0.05 & 1.92 & 0.019 & $\mathrm{~T}$ \\
\hline \multicolumn{5}{|l|}{ Formicidae } \\
\hline Atta mexicana & 0.02 & 1.92 & 0.019 & $\mathrm{~T}$ \\
\hline Camponotus atriceps & 0.19 & 3.85 & 0.039 & $\mathrm{~T}$ \\
\hline Nomamyrmex esenbecki & 0.15 & 7.69 & 0.077 & $\mathrm{~T}$ \\
\hline Pheidole sp. & 0.02 & 1.92 & 0.019 & $\mathrm{~T}$ \\
\hline Solenopsis gemminata & 0.18 & 5.77 & 0.058 & $\mathrm{~T}$ \\
\hline Sphecidae & 0.18 & 1.92 & 0.019 & $\mathrm{~T}$ \\
\hline Vespidae & 0.84 & 5.77 & 0.058 & $\mathrm{~T}$ \\
\hline \multicolumn{5}{|l|}{ Homópteros } \\
\hline Cicadeliidae & 1.50 & 36.54 & 0.365 & $\mathrm{~T}$ \\
\hline Cicadidae & 1.18 & 3.85 & 0.039 & $\mathrm{~T}$ \\
\hline Delphacidae & 0.04 & 3.85 & 0.039 & $\mathrm{~T}$ \\
\hline Penthiminae & 0.29 & 1.92 & 0.019 & $\mathrm{~T}$ \\
\hline \multicolumn{5}{|l|}{ Membracidae } \\
\hline Bilimekia sp. & 1.01 & 5.77 & 0.058 & $\mathrm{~T}$ \\
\hline \multicolumn{5}{|l|}{ Lepidopteros } \\
\hline Noctuidae & 17.76 & 61.54 & 0.615 & $\mathrm{~T}$ \\
\hline Indeterminados & 12.14 & 34.62 & 0.346 & $æ$ \\
\hline \multicolumn{5}{|l|}{ Megalópteros } \\
\hline \multicolumn{5}{|l|}{ Corydalidae } \\
\hline Corydalus texanus & 24.22 & 21.15 & 0.211 & Ж \\
\hline \multicolumn{5}{|l|}{ Odonatos } \\
\hline Aehsnidae (larvas) & 0.16 & 1.92 & 0.019 & A \\
\hline \multicolumn{5}{|l|}{ Coenagrionidae } \\
\hline Argia sp. (larvas) & 0.35 & 3.85 & 0.039 & A \\
\hline \multicolumn{5}{|l|}{ Gomphidae } \\
\hline Dromogompus sp. (larvas) & 0.88 & 1.92 & 0.019 & A \\
\hline Libellulidae & 1.72 & 9.62 & 0.096 & Ж \\
\hline Indeterminados & 0.43 & 1.92 & 0.019 & Ж \\
\hline \multicolumn{5}{|l|}{ Ortópteros } \\
\hline Acrididae & 0.09 & 1.92 & 0.019 & $\mathrm{~T}$ \\
\hline Blattidae & 0.49 & 5.77 & 0.05 & $\mathrm{~T}$ \\
\hline Gryllidae & 1.09 & 9.62 & 0.096 & $\mathrm{~T}$ \\
\hline
\end{tabular}


Concluye Cuadro 2

\begin{tabular}{|c|c|c|c|c|}
\hline Componentes alimentarios & PI (\%) & FO (\%) & IA & Hábito \\
\hline Indeterminados & 0.02 & 1.92 & 0.019 & $\mathrm{~T}$ \\
\hline \multicolumn{5}{|l|}{ Tricópteros } \\
\hline \multicolumn{5}{|l|}{ Hydropsychidae } \\
\hline Hydropsyche sp. (larvas) & 0.01 & 1.92 & 0.019 & A \\
\hline Leptonema sp. & 0.01 & 1.92 & 0.019 & $\mathrm{~T}$ \\
\hline \multicolumn{5}{|l|}{ Peces } \\
\hline \multicolumn{5}{|l|}{ Cichlidae } \\
\hline Cichlasoma nigrofasciatum & 0.24 & 1.92 & 0.019 & A \\
\hline \multicolumn{5}{|l|}{ Anfibios anuros } \\
\hline \multicolumn{5}{|l|}{ Ranidae } \\
\hline Lithobates sp. (larvas) & 0.93 & 1.92 & 0.019 & A \\
\hline Materia animal mixta indeterminada & 2.76 & 26.92 & 0.269 & \\
\hline Materia mineral (arena y piedras) & - & 5.77 & - & \\
\hline
\end{tabular}

\section{Cuadro 3}

Composición de la dieta de juveniles de Lithobates zweifeli de acuerdo con el porcentaje de ingestión (PI), frecuencia de ocurrencia (FO) e índice de Albertaine (IA). Los hábitos de las presas son: A= Acuático, $\mathrm{T}=$ Terrestre, $\mathbb{K}=$ Ambiguo y $æ=$ Desconocido.

\begin{tabular}{|c|c|c|c|c|}
\hline Componentes alimentarios & PI (\%) & FO (\%) & IA & Hábito \\
\hline Materia vegetal & - & 20.8 & - & \\
\hline \multicolumn{5}{|l|}{ Moluscos Gasterópodos } \\
\hline \multicolumn{5}{|l|}{ Lymnaeidae } \\
\hline Physa sp. & 0.11 & 4.17 & 0.042 & A \\
\hline Fossaria sp. & 0.24 & 4.17 & 0.042 & A \\
\hline \multicolumn{5}{|l|}{ Isópodos } \\
\hline Oniscidae & 0.50 & 4.17 & 0.042 & $\mathrm{~T}$ \\
\hline Quilópodos Geophilomorpha & 1.66 & 4.17 & 0.042 & $\mathrm{~T}$ \\
\hline \multicolumn{5}{|l|}{ Arácnidos } \\
\hline Lycosidae & 3.54 & 16.67 & 0.167 & $\mathrm{~T}$ \\
\hline Theridiidae & 0.06 & 4.17 & 0.042 & $\mathrm{~T}$ \\
\hline Zodariidae & 0.47 & 4.17 & 0.042 & $\mathrm{~T}$ \\
\hline \multicolumn{5}{|l|}{ Opiliones } \\
\hline \multicolumn{5}{|l|}{ Sclerosomatidae } \\
\hline Leiobonum sp. & 0.01 & 4.17 & 0.042 & $\mathrm{~T}$ \\
\hline
\end{tabular}


Continùa Cuadro 3

\begin{tabular}{|c|c|c|c|c|}
\hline Componentes alimentarios & PI (\%) & FO $(\%)$ & IA & Hábito \\
\hline \multicolumn{5}{|l|}{ Insectos } \\
\hline \multicolumn{5}{|l|}{ Coleópteros } \\
\hline Calendridae & 0.45 & 8.33 & 0.083 & $\mathrm{~T}$ \\
\hline Cantharidae & 0.30 & 8.33 & 0.083 & $\mathrm{~T}$ \\
\hline \multicolumn{5}{|l|}{ Carabidae } \\
\hline Selenophorus sp. & 0.27 & 4.17 & 0.042 & $\mathrm{~T}$ \\
\hline Stenomorphus sp. & 0.55 & 4.17 & 0.042 & $\mathrm{~T}$ \\
\hline Indeterminados & 1.71 & 16.67 & 0.167 & $\mathrm{~T}$ \\
\hline \multicolumn{5}{|l|}{ Cicindelidae } \\
\hline \multirow{2}{*}{\multicolumn{5}{|c|}{ Hidrophilidae }} \\
\hline & & & & \\
\hline Hydrophilus sp. (larvas) & 2.55 & 20.83 & 0.208 & A \\
\hline Lampiridae & 0.21 & 4.17 & 0.042 & $\mathrm{~T}$ \\
\hline \multicolumn{5}{|l|}{ Scarabeidae } \\
\hline Aphodius sp. & 0.08 & 8.33 & 0.083 & $\mathrm{~T}$ \\
\hline Indeterminados & 0.05 & 4.17 & 0.042 & $æ$ \\
\hline \multicolumn{5}{|l|}{ Dípteros } \\
\hline \multicolumn{5}{|l|}{ Caratopogonidae } \\
\hline Dasyhelea saltensis & 0.03 & 4.17 & 0.042 & $\mathrm{~T}$ \\
\hline \multicolumn{5}{|l|}{ Dolichopodidae } \\
\hline Dolichopus sp. & 0.07 & 4.17 & 0.042 & $\mathrm{~T}$ \\
\hline \multicolumn{5}{|l|}{ Lycoridae } \\
\hline Sciara sp. & 0.03 & 12.50 & 0.125 & $\mathrm{~T}$ \\
\hline \multicolumn{5}{|l|}{ Simuliideae } \\
\hline Simulium sp. & 9.02 & 20.83 & 0.208 & A \\
\hline \multicolumn{5}{|l|}{ Stratiomyidae } \\
\hline Stratiomys sp. & 0.42 & 4.17 & 0.042 & $\mathrm{~T}$ \\
\hline \multicolumn{5}{|l|}{ Tipulidae } \\
\hline Tipula sp. & 1.39 & 12.50 & 0.125 & A \\
\hline \multicolumn{5}{|l|}{ Efemerópteros } \\
\hline \multicolumn{5}{|l|}{ Baetidae } \\
\hline Camelobaetidius sp. & 0.07 & 4.17 & 0.042 & A \\
\hline \multicolumn{5}{|l|}{ Hemípteros } \\
\hline \multicolumn{5}{|l|}{ Aetalionidae } \\
\hline Aetalion sp. & 0.24 & 12.50 & 0.125 & $\mathrm{~T}$ \\
\hline Coreidae & 1.43 & 4.17 & 0.042 & $\mathrm{~T}$ \\
\hline Miridae & 0.04 & 4.17 & 0.042 & $\mathrm{~T}$ \\
\hline \multicolumn{5}{|l|}{ Nepidae } \\
\hline Ranatra sp. & 0.68 & 8.33 & 0.083 & A \\
\hline Pentatomidae & 0.13 & 4.17 & 0.042 & $\mathrm{~T}$ \\
\hline \multicolumn{5}{|l|}{ Veliidae } \\
\hline Microvelia sp. & 0.05 & 4.17 & 0.042 & A \\
\hline Rhagovelia sp. & 0.04 & 4.17 & 0.042 & $\mathrm{~T}$ \\
\hline
\end{tabular}


Concluye Cuadro 3

\begin{tabular}{|c|c|c|c|c|}
\hline Componentes alimentarios & PI (\%) & FO (\%) & IA & Hábito \\
\hline Indeterminados & 1.79 & 4.17 & 0.042 & $æ$ \\
\hline \multicolumn{5}{|l|}{ Himenópteros } \\
\hline Figitidae & 0.12 & 4.17 & 0.042 & $\mathrm{~T}$ \\
\hline \multicolumn{5}{|l|}{ Formicidae } \\
\hline Camponotus atriceps & 0.51 & 8.33 & 0.083 & $\mathrm{~T}$ \\
\hline Nomamyrmex esenbecki & 0.13 & 8.33 & 0.083 & $\mathrm{~T}$ \\
\hline Pheidole sp. & 0.05 & 4.17 & 0.042 & $\mathrm{~T}$ \\
\hline Solenopsis gemminata & 0.06 & 4.17 & 0.042 & $\mathrm{~T}$ \\
\hline Sphecidae & 0.46 & 4.17 & 0.042 & $\mathrm{~T}$ \\
\hline \multicolumn{5}{|l|}{ Homópteros } \\
\hline Cicadellidae & 2.48 & 45.83 & 0.458 & $\mathrm{~T}$ \\
\hline Penthimiinae & 0.74 & 4.17 & 0.042 & $\mathrm{~T}$ \\
\hline Cicadidae & 0.26 & 4.17 & 0.042 & $\mathrm{~T}$ \\
\hline \multicolumn{5}{|l|}{ Membracidae } \\
\hline Bilimekia $\mathrm{sp}$. & 2.58 & 12.50 & 0.125 & $\mathrm{~T}$ \\
\hline \multicolumn{5}{|l|}{ Lepidópteros } \\
\hline Noctuidae & 34.26 & 83.33 & 0.833 & $\mathrm{~T}$ \\
\hline Indeterminados & 24.88 & 45.83 & 0.458 & $æ$ \\
\hline \multicolumn{5}{|l|}{ Odonatos } \\
\hline \multicolumn{5}{|l|}{ Coenagrionidae } \\
\hline Argia sp. (larvas) & 0.47 & 4.17 & 0.042 & A \\
\hline Libellulidae & 0.42 & 12.50 & 0.125 & Ж \\
\hline Indeterminados & 1.11 & 4.17 & 0.042 & Ж \\
\hline \multicolumn{5}{|l|}{ Ortopteros } \\
\hline Gryllidae & 0.09 & 4.17 & 0.042 & $\mathrm{~T}$ \\
\hline Materia animal mixta indeterminada & 1.22 & 16.67 & 0.167 & $æ$ \\
\hline Materia mineral (piedras) & - & 12.5 & - & \\
\hline
\end{tabular}


Mendoza Estrada et al.: Dieta de Lithobates sweifeli Hillis, Frost y Webb 1984 (Anura: Ranidae)

\section{Cuadro 4}

Composición de la dieta de hembras de Lithobates zweifeli de acuerdo con el porcentaje de ingestión (PI), frecuencia de ocurrencia (FO) e índice de Albertaine (IA). Los hábitos de las presas son: $\mathrm{A}=$ acuático, $\mathrm{T}=$ terrestre, $\mathbb{K}=$ ambiguo y $æ=$ desconocido.

\begin{tabular}{|c|c|c|c|c|}
\hline Componentes alimentarios & PI (\%) & FO $(\%)$ & IA & Hábito \\
\hline Materia vegetal & - & 41.6 & - & \\
\hline \multicolumn{5}{|l|}{ Anélidos } \\
\hline Oligochaeta & 6.34 & 16.67 & 0.167 & $\mathrm{~T}$ \\
\hline \multicolumn{5}{|l|}{ Moluscos Gasterópodos } \\
\hline \multicolumn{5}{|l|}{ Planorbidae } \\
\hline Helisoma sp. & 1.82 & 4.17 & 0.042 & A \\
\hline Quilópodos Geophilomorpha & 0.29 & 4.17 & 0.042 & $\mathrm{~T}$ \\
\hline \multicolumn{5}{|l|}{ Isópodos } \\
\hline Oniscidae & 0.35 & 4.17 & 0.042 & $\mathrm{~T}$ \\
\hline \multicolumn{5}{|l|}{ Arácnidos } \\
\hline \multicolumn{5}{|l|}{ Buthidae } \\
\hline Centruroides l. limpidus & 5.76 & 8.33 & 0.083 & $\mathrm{~T}$ \\
\hline Dipluridae & 0.1 & 8.33 & 0.083 & $\mathrm{~T}$ \\
\hline Dysderidae & 0.07 & 4.17 & 0.042 & $\mathrm{~T}$ \\
\hline Lycosidae & 4.8 & 37.5 & 0.375 & $\mathrm{~T}$ \\
\hline Pholcidae & 0.02 & 4.17 & 0.042 & $\mathrm{~T}$ \\
\hline Salticidae & 0.04 & 4.17 & 0.042 & $\mathrm{~T}$ \\
\hline Theridiidae & 0.02 & 4.17 & 0.042 & $\mathrm{~T}$ \\
\hline Uloboridae & 0.13 & 4.17 & 0.042 & $\mathrm{~T}$ \\
\hline Indeterminados & 0.07 & 8.33 & 0.083 & $\mathrm{~T}$ \\
\hline \multicolumn{5}{|l|}{ Opiliones } \\
\hline \multicolumn{5}{|l|}{ Sclerosomatidae } \\
\hline Leiobonum sp. & 2.64 & 16.67 & 0.167 & $\mathrm{~T}$ \\
\hline \multicolumn{5}{|l|}{ Insectos } \\
\hline \multicolumn{5}{|l|}{ Coleópteros } \\
\hline Cantharidae & 0.37 & 4.17 & 0.042 & $\mathrm{~T}$ \\
\hline Carabidae & 0.84 & 12.5 & 0.125 & $\mathrm{~T}$ \\
\hline \multicolumn{5}{|l|}{ Cicindelidae } \\
\hline Cicindella sp. & 0.12 & 4.17 & 0.042 & $\mathrm{~T}$ \\
\hline Elateridae & 0.16 & 4.17 & 0.042 & $\mathrm{~T}$ \\
\hline \multicolumn{5}{|l|}{ Hydrophilidae } \\
\hline Hydrophilus sp. (larvas) & 1.0 & 12.5 & 0.125 & A \\
\hline Lampiridae & 0.02 & 4.17 & 0.042 & $\mathrm{~T}$ \\
\hline \multicolumn{5}{|l|}{ Melolonthidae } \\
\hline Cyclocephala sp. & 0.61 & 4.17 & 0.042 & $\mathrm{~T}$ \\
\hline
\end{tabular}


Continúa Cuadro 4

\begin{tabular}{|c|c|c|c|c|}
\hline Componentes alimentarios & PI (\%) & FO $(\%)$ & IA & Hábito \\
\hline Staphilinidae & 0.02 & 4.17 & 0.042 & $\mathrm{~T}$ \\
\hline Indeterminados & 0.07 & 8.33 & 0.083 & $æ$ \\
\hline \multicolumn{5}{|l|}{ Dermapteros } \\
\hline \multicolumn{5}{|l|}{ Forficulidae } \\
\hline Doru lineare & 1.22 & 12.5 & 0.125 & A \\
\hline \multicolumn{5}{|l|}{ Dípteros } \\
\hline Chironomidae (larvas) & 0.02 & 4.17 & 0.042 & A \\
\hline \multicolumn{5}{|l|}{ Ceratopogonidae } \\
\hline Dasyhelea saltensis & 0.31 & 4.17 & 0.042 & $\mathrm{~T}$ \\
\hline \multicolumn{5}{|l|}{ Drosophilidae } \\
\hline $\begin{array}{l}\text { Drosophila sp. } \\
\text { Simulidae }\end{array}$ & 0.05 & 4.17 & 0.042 & $\mathrm{~T}$ \\
\hline Simulium sp. & 7.34 & 8.33 & 0.083 & A \\
\hline \multicolumn{5}{|l|}{ Stratiomyidae } \\
\hline Stratiomys sp. & 0.01 & 4.17 & 0.042 & $\mathrm{~T}$ \\
\hline \multicolumn{5}{|l|}{ Efemerópteros } \\
\hline \multicolumn{5}{|l|}{ Baetidae } \\
\hline Baetodes sp. & 0.01 & 4.17 & 0.042 & A \\
\hline \multicolumn{5}{|l|}{ Leptohyphidae } \\
\hline Tricorythodes sp. & 0.02 & 4.17 & 0.042 & A \\
\hline \multicolumn{5}{|l|}{ Hemípteros } \\
\hline \multicolumn{5}{|l|}{ Aetalionidae } \\
\hline Aetalion sp. & 0.21 & 8.33 & 0.083 & $\mathrm{~T}$ \\
\hline Corixidae & 1.07 & 4.17 & 0.042 & $\mathrm{~T}$ \\
\hline \multicolumn{5}{|l|}{ Gelastocoridae } \\
\hline Nerthra sp. & 0.81 & 8.33 & 0.083 & $\mathrm{~A}$ \\
\hline Lygaeidae & 0.86 & 20.83 & 0.208 & $\mathrm{~T}$ \\
\hline Reduvidae & 0.29 & 12.5 & 0.125 & $\mathrm{~T}$ \\
\hline Indeterminados & 0.03 & 4.17 & 0.042 & $æ$ \\
\hline \multicolumn{5}{|l|}{ Himenópteros } \\
\hline Adrenidae & 0.16 & 8.33 & 0.083 & $\mathrm{~T}$ \\
\hline Chalcididae & 0.09 & 4.17 & 0.042 & $\mathrm{~T}$ \\
\hline \multicolumn{5}{|l|}{ Formicidae } \\
\hline Atta mexicana & 0.25 & 8.33 & 0.083 & $\mathrm{~T}$ \\
\hline Camponotus atriceps & 0.41 & 12.5 & 0.125 & $\mathrm{~T}$ \\
\hline Nomamyrmex esenbecki & 0.14 & 4.17 & 0.042 & $\mathrm{~T}$ \\
\hline Solenopsis gemminata & 0.27 & 4.17 & 0.042 & $\mathrm{~T}$ \\
\hline Indeterminados & 0.01 & 4.17 & 0.042 & $\mathrm{~T}$ \\
\hline \multicolumn{5}{|l|}{ Homópteros } \\
\hline Cicadellidae & 0.41 & 20.83 & 0.208 & $\mathrm{~T}$ \\
\hline Typhlocibinae & 0.15 & 4.17 & 0.042 & $\mathrm{~T}$ \\
\hline Cicadidae & 2.13 & 12.5 & 0.125 & $\mathrm{~T}$ \\
\hline
\end{tabular}


Mendoza Estrada et al.: Dieta de Lithobates sweifeli Hillis, Frost y Webb 1984 (Anura: Ranidae)

\section{Concluye Cuadro 4}

\begin{tabular}{|c|c|c|c|c|}
\hline Componentes alimentarios & PI (\%) & FO $(\%)$ & IA & Hábito \\
\hline \multicolumn{5}{|l|}{ Lepidópteros } \\
\hline Noctuidae & 4.73 & 33.33 & 0.333 & $\mathrm{~T}$ \\
\hline Indeterminados & 0.67 & 12.5 & 0.125 & $\mathfrak{x}$ \\
\hline \multicolumn{5}{|l|}{ Megalopteros } \\
\hline \multicolumn{5}{|l|}{ Corydalidae } \\
\hline Corydalus texanus & 39.3 & 41.67 & 0.417 & Ж \\
\hline \multicolumn{5}{|l|}{ Odonatos } \\
\hline Aeshnidae & 0.27 & 4.17 & 0.042 & 五 \\
\hline \multicolumn{5}{|l|}{ Gomphidae } \\
\hline Dromogomphus sp. & 1.46 & 4.17 & 0.042 & A \\
\hline Libellulidae & 2.84 & 12.5 & 0.125 & Ж \\
\hline \multicolumn{5}{|l|}{ Ortópteros } \\
\hline Acrididae & 0.15 & 4.17 & 0.042 & $\mathrm{~T}$ \\
\hline Blattidae & 0.15 & 4.17 & 0.042 & $\mathrm{~T}$ \\
\hline Gryllidae & 1.4 & 8.33 & 0.083 & $\mathrm{~T}$ \\
\hline Indeterminados & 0.25 & 4.17 & 0.042 & $\mathrm{~T}$ \\
\hline \multicolumn{5}{|l|}{ Tricópteros } \\
\hline \multicolumn{5}{|l|}{ Hydropsychidae } \\
\hline Hydopsyche sp. & 0.02 & 4.17 & 0.042 & A \\
\hline Leptonema sp. & 0.02 & 8.33 & 0.083 & $\mathrm{~T}$ \\
\hline \multicolumn{5}{|l|}{ Odontoceridae } \\
\hline Marilia sp. & 0.19 & 4.17 & 0.042 & $\mathrm{~T}$ \\
\hline \multicolumn{5}{|l|}{ Peces } \\
\hline \multicolumn{5}{|l|}{ Cichlidae } \\
\hline Cichlasoma nigrofasciatum & 0.39 & 4.17 & 0.042 & $\mathrm{~A}$ \\
\hline Indeterminados & 0.13 & 4.17 & 0.042 & A \\
\hline \multicolumn{5}{|l|}{ Anfibios anuros } \\
\hline \multicolumn{5}{|l|}{ Ranidae } \\
\hline Lithobates sp. (larvas) & 3.15 & 7.14 & 0.071 & $\mathrm{~A}$ \\
\hline Materia animal mixta indeterminada & 3.0 & 33.33 & 0.333 & $æ$ \\
\hline Materia mineral (arena y piedras) & - & 4.17 & - & \\
\hline
\end{tabular}




\section{Cuadro 5}

Composición de la dieta de machos de Lithobates zweifeli de acuerdo con el porcentaje de ingestión (PI), frecuencia de ocurrencia (FO) e índice de Albertaine (IA). Los hábitos de las presas son: $\mathrm{A}=$

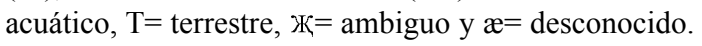

\begin{tabular}{|c|c|c|c|c|}
\hline Componentes alimentarios & PI (\%) & FO (\%) & $\mathbf{I A}$ & Hábito \\
\hline Materia vegetal & - & 4.17 & - & \\
\hline \multicolumn{5}{|l|}{ Moluscos Gasterópodos } \\
\hline \multicolumn{5}{|l|}{ Lymnaeidae } \\
\hline Physa sp. & 0.17 & 4.17 & 0.042 & A \\
\hline \multicolumn{5}{|l|}{ Isópodos } \\
\hline Oniscidae & 1.93 & 4.17 & 0.042 & $\mathrm{~T}$ \\
\hline \multicolumn{5}{|l|}{ Arácnidos } \\
\hline Amaurobiidae & 0.09 & 4.17 & 0.042 & $\mathrm{~T}$ \\
\hline \multicolumn{5}{|l|}{ Buthidae } \\
\hline Centruroides l. limpidus & 0.77 & 4.17 & 0.042 & $\mathrm{~T}$ \\
\hline Lycosidae & 3.72 & 20.8 & 0.208 & $\mathrm{~T}$ \\
\hline Theridiidae & 0.10 & 4.17 & 0.042 & $\mathrm{~T}$ \\
\hline Tretagnathidae & 0.56 & 4.17 & 0.042 & $\mathrm{~T}$ \\
\hline \multicolumn{5}{|l|}{ Opiliones } \\
\hline \multicolumn{5}{|l|}{ Sclerosomatidae } \\
\hline Leiobonum sp. & 2.54 & 20.8 & 0.208 & $\mathrm{~T}$ \\
\hline \multicolumn{5}{|l|}{ Insectos } \\
\hline \multicolumn{5}{|l|}{ Coleópteros } \\
\hline Blattidae & 1.07 & 4.17 & 0.042 & $\mathrm{~T}$ \\
\hline Carabidae & 0.17 & 4.17 & 0.042 & $\mathrm{~T}$ \\
\hline \multicolumn{5}{|l|}{ Cicindelidae } \\
\hline Cicindella sp. & 1.98 & 8.33 & 0.083 & $\mathrm{~T}$ \\
\hline \multicolumn{5}{|l|}{ Curculionidae } \\
\hline Auleutes sp. & 0.19 & 4.17 & 0.042 & A \\
\hline Elateridae & 0.87 & 12.5 & 0.125 & $\mathrm{~T}$ \\
\hline \multicolumn{5}{|l|}{ Hydrophilidae } \\
\hline Hydrophilus sp. & 0.46 & 4.17 & 0.042 & A \\
\hline \multicolumn{5}{|l|}{ Melolonthidae } \\
\hline Cyclocephala sp. & 1.90 & 4.17 & 0.042 & $\mathrm{~T}$ \\
\hline Diplotaxis sp. & 0.23 & 4.17 & 0.042 & $\mathrm{~T}$ \\
\hline Rodobaenus sp. & 0.24 & 4.17 & 0.042 & $\mathrm{~T}$ \\
\hline Staphilinidae & 0.06 & 4.17 & 0.042 & $\mathrm{~T}$ \\
\hline Tenebrionidae & 0.79 & 8.33 & 0.083 & $\mathrm{~T}$ \\
\hline Indeterminados & 0.32 & 12.5 & 0.125 & $æ$ \\
\hline \multicolumn{5}{|l|}{ Colémbolos } \\
\hline \multicolumn{5}{|l|}{ Sminthurididae } \\
\hline Sminthurides sp. & 0.05 & 8.33 & 0.083 & A \\
\hline
\end{tabular}


Continúa Cuadro 5

\begin{tabular}{|c|c|c|c|c|}
\hline Componentes alimentarios & PI (\%) & FO $(\%)$ & IA & Hábito \\
\hline \multicolumn{5}{|l|}{ Dermápteros } \\
\hline \multicolumn{5}{|l|}{ Forficulidae } \\
\hline Doru lineare & 1.89 & 12.5 & 0.125 & $\mathrm{~T}$ \\
\hline \multicolumn{5}{|l|}{ Dípteros } \\
\hline \multicolumn{5}{|l|}{ Ceratopogonidae } \\
\hline Dasyhelea saltensis & 2.15 & 4.17 & 0.042 & $\mathrm{~T}$ \\
\hline Chironomidae & 0.80 & 4.17 & 0.042 & A \\
\hline \multicolumn{5}{|l|}{ Dolichopodidae } \\
\hline Dolichopus sp. & 0.04 & 4.17 & 0.042 & $\mathrm{~T}$ \\
\hline \multicolumn{5}{|l|}{ Drosophilidae } \\
\hline Drosophila sp. & 0.07 & 4.17 & 0.042 & $\mathrm{~T}$ \\
\hline \multicolumn{5}{|l|}{ Sciaridae } \\
\hline Sciara sp. & 0.03 & 4.17 & 0.042 & $\mathrm{~T}$ \\
\hline \multicolumn{5}{|l|}{ Simulidae } \\
\hline Simulium sp. & 2.92 & 12.5 & 0.125 & A \\
\hline \multicolumn{5}{|l|}{ Stratiomyidae } \\
\hline Stratiomys sp. & 0.11 & 4.17 & 0.042 & $\mathrm{~T}$ \\
\hline \multicolumn{5}{|l|}{ Tipulidae } \\
\hline Tipula sp. & 0.43 & 8.33 & 0.083 & A \\
\hline \multicolumn{5}{|l|}{ Efemerópteros } \\
\hline \multicolumn{5}{|l|}{ Baetidae } \\
\hline Baetodes sp. & 0.06 & 4.17 & 0.042 & A \\
\hline \multicolumn{5}{|l|}{ Leptohyphidae } \\
\hline Leptohyphes sp. & 0.03 & 4.17 & 0.042 & A \\
\hline \multicolumn{5}{|l|}{ Anthocoridae } \\
\hline Anthocoris sp. & 0.10 & 4.17 & 0.042 & $\mathrm{~T}$ \\
\hline \multicolumn{5}{|l|}{ Hemípteros } \\
\hline Coreidae & 2.01 & 8.33 & 0.083 & $\mathrm{~T}$ \\
\hline \multicolumn{5}{|l|}{ Gelastocoridae } \\
\hline Nerthra sp. & 2.88 & 8.33 & 0.083 & A \\
\hline Lygaeidae & 3.34 & 25.0 & 0.250 & $\mathrm{~T}$ \\
\hline Nabidae & 0.09 & 4.17 & 0.042 & $\mathrm{~T}$ \\
\hline Indeterminados & 0.10 & 8.33 & 0.083 & $æ$ \\
\hline \multicolumn{5}{|l|}{ Homópteros } \\
\hline Cicadellidae & 0.96 & 20.8 & 0.208 & $\mathrm{~T}$ \\
\hline Cicadidae & 1.06 & 8.33 & 0.083 & $\mathrm{~T}$ \\
\hline Delphacidae & 0.13 & 8.33 & 0.083 & $\mathrm{~T}$ \\
\hline \multicolumn{5}{|l|}{ Himenópteros } \\
\hline \multicolumn{5}{|l|}{ Formicidae } \\
\hline Camponotus atriceps & 0.56 & 4.17 & 0.042 & $\mathrm{~T}$ \\
\hline Nomamyrmex esenbecki & 0.04 & 4.17 & 0.042 & $\mathrm{~T}$ \\
\hline Solenopsis gemminata & 0.01 & 4.17 & 0.042 & $\mathrm{~T}$ \\
\hline Vespidae & 2.61 & 12.5 & 0.125 & $\mathrm{~T}$ \\
\hline
\end{tabular}


Concluye Cuadro 5

\begin{tabular}{|c|c|c|c|c|}
\hline Componentes alimentarios & PI (\%) & FO (\%) & IA & Hábito \\
\hline \multicolumn{5}{|l|}{ Lepidópteros } \\
\hline Noctuidae & 19.36 & 45.8 & 0.458 & $\mathrm{~T}$ \\
\hline Indeterminados & 1.12 & 4.17 & 0.042 & $æ$ \\
\hline \multicolumn{5}{|l|}{ Megalopteros } \\
\hline \multicolumn{5}{|l|}{ Corydalidae } \\
\hline Corydalus texanus & 5.99 & 8.33 & 0.083 & 五 \\
\hline \multicolumn{5}{|l|}{ Odonatos } \\
\hline Aehsnidae & 3.67 & 4.17 & 0.042 & 术 \\
\hline \multicolumn{5}{|l|}{ Coenagrionidae } \\
\hline Argia sp. & 0.50 & 4.17 & 0.042 & A \\
\hline Indeterminados & 5.87 & 4.17 & 0.042 & 五 \\
\hline \multicolumn{5}{|l|}{ Ortópteros } \\
\hline Gryllidae & 2.49 & 20.8 & 0.208 & $\mathrm{~T}$ \\
\hline Indeterminados & 1.65 & 8.33 & 0.083 & $\mathrm{~T}$ \\
\hline \multicolumn{5}{|l|}{ Tricópteros } \\
\hline Hydroptilidae & 0.32 & 4.17 & 0.042 & $\mathrm{~T}$ \\
\hline \multicolumn{5}{|l|}{ Anfibios anuros } \\
\hline \multicolumn{5}{|l|}{ Ranidae } \\
\hline Lithobates sp. (larvas) 1 & 3.75 & 8.33 & 0.083 & A \\
\hline Materia animal mixta indeterminada & 4.65 & 29.2 & 0.292 & $\mathfrak{x}$ \\
\hline Materia mineral (piedras) & - & 25.0 & - & \\
\hline
\end{tabular}

\section{DISCUSIÓN}

Lithobates zweifeli es una rana de hábitos nocturnos que consume principalmente insectos. Pequeños vertebrados como peces de la familia Cichlidae y renacuajos de Lithobates sp., aunque no forman parte usual de la dieta, fueron ingeridos ocasionalmente por los adultos, como ha sido observado en L. catesbianus en Oklahoma (Tyler \& Hoestenbach 1979). Casos bien documentados de canibalismo en el género Litohobates también han sido registrados en L. catesbianus (Werner et. al. 1995, Beard \& Baillie 1998 y Daza-Vaca \& Castro-Herrera 1999) y L. aurora draytoni (Hayes \& Tennant 1985).

La mayor parte de la dieta, de machos, hembras y juveniles de L. zweifeli, en las épocas de estiaje y de lluvias, estuvo formada por presas de hábitos terrestres, lo que revela que tiene hábitos riparios similares a los de L. brownorum que se alimenta en micro ambientes terrestres (Ramírez-Bautista \& Lemos-Espinal 2004) y contrasta con los hábitos típicamente acuáticos de L. vaillanti (Ramírez-Bautista \& Lemos-Espinal 2004) y L. montezumae (Rodríguez-Blanco 1990). Aunque 
durante el período de lluvias el número de componentes alimentarios ingeridos por L. zweifeli fue mayor que en la época seca, no hubo diferencias estadísticamente significativas entre los dos períodos. Insectos de las familias Noctuidae (Lepidoptera), Corydalidae (Megaloptera), Sclerosomatidae (Opiliones) y Simuliidae (Diptera) fueron los grupos consumidos en mayor cantidad a lo largo del año por adultos y juveniles. Sin embargo, los lepidópteros de la familia Noctuidae, de acuerdo con los valores del IA, fueron el alimento preferido en el período de lluvias. Litohobates brownorum, congénere de hábitos terrestres en un ambiente tropical consume en mayor cantidad coleópteros, diplópodos, lepidópteros e himenópteros. L. vaillanti de hábitos más acuáticos consume decápodos (crustáceos), stylomatóforos (moluscos) y homópteros (RamírezBautista \& Lemos-Espinal 2004), mientras que L. montezumae de hábitos acuáticos en ambientes semifríos, consume decápodos (crustáceos), moluscos y cantidades importantes de materia vegetal. Es claro que la composición y consumo de alimento varía entre las distintas especies, áreas y micro ambientes, lo que revela que las ranas del género Lithobates son generalistas, y pueden consumir una gama de alimento que varía desde presas totalmente acuáticas hasta especies terrestres voladoras como lepidópteros. Lithobates zweifeli también consume escorpiones (Centruroides l. limpidus), a los que parece ser inmune como se observó en este estudio (ver Anexo 1). De hecho, otro congénere, L. catesbianus, es inmune a la bufonina (Taylor \& Michael 1971, Brown 1974, Platt \& Fontenot 1993, Stuart 1995, Daza-Vaca \& Castro-Herrera 1999).

Los hábitos de alimentación de tipo generalista en las ranas (Forstner et. al. 1998, Hayes \& Tennant 1985) y la ausencia de especialización sobre un tipo particular de alimento aun en la época de lluvias, contribuyen a explicar porque no existe traslape de los nichos tróficos entre los sexos en L. zweifeli. Es cierto que los individuos pequeños de esta especie tienen poca habilidad para obtener alimento y menor acceso a presas de tamaño grande, como lo reveló el número de componentes del alimento; sin embargo, la estacionalidad y la presencia de agua en el río durante todo el año en el área de estudio, favorece la disponibilidad de presas de distintos tamaños para la población de L. zweifeli que ahí se desarrolla. La materia vegetal fue encontrada con frecuencia en los estómagos de los individuos examinados, y aunque usualmente ha sido atribuida a la ingestión accidental en varias especies de ranas (Hayes \& Tennant 1985, Rodríguez-Blanco 1990, Borczyk 2001) también se ha sugerido que funciona como complemento en la dieta (Brooks 1964, Das 1996). Proponemos que L. zweifeli ingiere materia vegetal para facilitar el desarrollo de simbiontes que contribuyen a la fermentación del alimento en el tubo digestivo de manera similar a como ocurre en reptiles herbívoros (Iverson 1982, da Silva et al. 1989). 


\section{AGRADECIMIENTOS}

Alfredo Chávez Martínez del Laboratorio de Herpetología del Centro de Investigaciones Biológicas (CIB) colaboró en los trabajos de campo. Juan Carlos Sandoval Manríquez, Armando Burgos Solorio, Ismael Anaya Calvo, Guadalupe Peña Chora y Adriana Trejo Loyo del Laboratorio de Parasitología Vegetal del CIB, nos apoyaron en la determinación de los contenidos estomacales y proporcionaron espacio físico para desarrollar esa parte del proyecto. Patricia Trujillo Jiménez del Laboratorio de Ictiología del CIB realizó comentarios y correcciones en versiones tempranas de este manuscrito. Dos revisiones anónimos contribuyeron a mejorar el contenido de este trabajo. A todos nuestros agradecimiento por su apoyo.

\section{LITERATURA CITADA}

Albertaine, B. J. 1973. Biologie des stades juveniles de Teleosteens Mugilidae: Mugil auratus Riso 1810, Mugil saliens Riso 1810. IH Régimen alimentaire. Aquaculture 2:251566.

Anderson, A., D. Haukos \& J. Anderson. 1999. Diet composition of three anurans from the Playa Wetlands of Northwest Texas. Copeia 1999(2):513-520.

Beard, K. H. \& J. Baillie. 1998. Rana catesbeiana. Diet. Herpetol. Review 29(1):40.

Borczyk, B. 2001. Rana temporaria. Diet. Herpetol. Review 32(3):184.

Brooks, G. R. Jr. 1964. An analysis of the food habits of the bullfrog Rana catesbeiana, by body size, sex, month and habitat. Virginia J. Science 15:175-186.

Brown, L. E. 1974. Behavioral reactions of bullfrogs while attempting to eat toads. Southwestern Nat. 19:335-336.

Castro-Franco, R., G. G. Vergara, G., M. G. Bustos Z. \& W. M. Arizmendi. 2006. Diversidad y distribución de anfibios del estado de Morelos, México. Acta Zool. Mex. (n.s.) 22(1):103-117.

Cloudsley-Thompson, J. 1999. The diversity of amphibians and reptiles. Springer. New York. 254 pp.

Cogalniceanu, D., W. Palmer \& C. Ciubuc. 2001. Feeding in anuran communities in the Danube floodplain. Amphibia-Reptilia 22:1-19.

Das, I. 1996. Folivory and seasonal changes in diet in Rana hexadactyla (Anura, Ranidae). J. Zool. (Lond.) 238:785-794.

da Silva, H R., M. C.de Britto-Pereira \& U. Caramaschi. 1989. Frugivory and seed dispersal by Hyla truncata, a neotropical treefrog. Copeia 1989(3):781-783.

da Silva, H. R. \& M. C. de Britto-Pereira. 2006. How much fruit do fruit-eating frogs eat? An investigation on the diet of Xenohyla truncata (Lissamphibia: Anura: Hylidae). $J$. Zool. (Lond.) 270:692-698.

Daza-Vaca,J. \& F. Castro-Herrera. 1999. Hábitos alimenticios de la rana toro (Rana catesbeiana) Anura: Ranidae en el Valle del Cauca, Colombia. Rev. Acad. Col. Ciencias XXIII (suplemento especial):265-274.

Duellman,W. E. \& L. Trueb. 1994. Biology of the amphibians. Johns Hopkins University Press, New York. 670 pp. 
Evans,M. \& M. Lampo. 1996. Diet of Bufo marinus in Venezuela. J. Herpetol. 30(1):73-76.

Forstner, J. M., M. R. J. Forstner \& J. R. Dixon. 1998. Ontogenetic effects on prey selection and food habits of two sympatric east Texas ranids: the southern leopard frog, Rana sphenocephala, and the bronze frog, Rana clamitans clamitans. Herpetol. Review 29(4): 208-211.

García, E. 1988. Modificaciones al sistema de clasificación climática de Köppen. Offset Larios, S.A. México, D. F. 217 pp.

Hayes, M. \& M. Tennant. 1985. Diet and feeding behavior of the California red-legged frog Rana aurora draytonii (Ranidae). Southwestern Nat. 30(4):601-605.

Hillis, D. M., J. S. Frost \& R. G. Webb. 1984. A new especies of frog of the Rana tarahumarae group from Southwestern Mexico. Copeia 1984(2):398-403.

Hirai, T. \& M. Matsui. 1999. Feeding habits of the pond frog, Rana nigromaculata, inhabiting rice fields in Kyoto, Japan. Copeia 1999(4):940-947.

Hulbert, S. H. 1978. The measurement of niche overlap and some relatives. Ecology 59:67-77.

Lagler, K. F. 1977. Freshwater fishery biology. Wm. C. Brown Company Pub. Dubuque, Iowa. $421 \mathrm{pp}$.

Legler, J. \& L. Sullivan. 1979. The application of stomach flushing to lizards and anurans. Herpetologica 35(2):107-110.

Levins, R. 1968. Evolution in changing environments: Some theoretical explorations. Princeton University Press, Princeton, New Jersey. 120 pp.

MacArthur, R. H. \& R. Levins. 1967. The limiting similarity, convergent, and divergent of coexisting species. Amer. Natl. 101:377-385.

Platt, S. G. \& L. W. Fontenot .1993. Bullfrog (Rana catesbeiana) predation on Gulf Coast toads (Bufo valliceps) in Louisiana. Bull. Chicago Herpet. Soc. 28:189-190.

Ramírez-Bautista, A. \& J. A. Lemos-Espinal. 2004. Diets of two syntopic populations of frogs, Rana vaillanti and Rana brownorum, from a tropical rain forest in Southern Veracruz, México. Southwestern Nat. 49(3):316-320.

Rodríguez-Blanco, M. 1990. Ciclos reproductivo y alimentario de Rana montezumae Baird, 1854 (Amphibia: Anura) del lago de Tecocomulco, Hidalgo, México. Bol. Soc. Herpetol. Mex. 2(2):6-10.

Siegel, S. 1976. Estadistica no paramétrica aplicada a las ciencias de la conducta. Editorial Trillas. México. 346 pp.

Stuart, J. N. 1995. Rana catesbeiana. Diet. Herpetol. Review 26(1):33.

Taylor, R. J. \& E. D. Michael.1971. Habitat effects on monthly foods of bullfrogs in eastern Texas. Proc. Conf. Southeastern Assoc. Gamme Fish Comm. 25:176-186.

Tyler, J. D. \& R. D. Hoestenbach, Jr. 1979. Differences in food of bullfrogs (Rana catesbeiana) from pond and stream habitat in Southwestern Oklahoma. Southwestern Nat. 24(1):33-38.

Van Sluys, M., D. Rocha \& B. Souza. 2001. Diet, reproduction and density of the Leptodactylid litter frog Zachaenus parvulus in an Atlantic rain forest of southeastern Brazil. J. Herpetol. 35(2):322-325. 
Werner E., G. Wellborn \& M. McPeek. 1995. Diet composition in postmetamorphic bullfrogs and green frogs: Implication for interspecific predation and competition. $J$. Herpetol. 29(4):600-607.

Windell, J. T. \& S. Bowen, H. 1978. Study of fish diets based on analysis of stomach contents. Pp. 219-226. In: T. Bagenal (ed). 1978. Methods assessment of fish production in freshwater. IBP Handbook No.3, Blackwell Scientific Publications Oxford.

Zaret, M. \& A. Rand. 1971. Competition in tropical stream fishes, support for the competitive exclusion principle. Ecology 52(2): 336-342. 
Mendoza Estrada et al.: Dieta de Lithobates sweifeli Hillis, Frost y Webb 1984 (Anura: Ranidae)

\section{Anexo 1}

Composición general de la dieta de Lithobates zweifeli de acuerdo con el porcentaje de ingestión (PI), frecuencia de ocurrencia (FO) e índice de Albertaine (IA). Los hábitos de las presas son: A= acuático, $\mathrm{T}=$ terrestre, $\aleph=$ ambiguo $\mathrm{y} æ=$ desconocido.

\begin{tabular}{|c|c|c|c|c|}
\hline Componentes alimentarios & PI (\%) & FO $(\%)$ & IA & Hábito \\
\hline Materia Vegetal & - & 29.16 & - & \\
\hline \multicolumn{5}{|l|}{ Anélidos } \\
\hline Oligochaeta & 2.89 & 5.56 & 0.056 & $\mathrm{~T}$ \\
\hline \multicolumn{5}{|l|}{ Moluscos Gasterópodos } \\
\hline \multicolumn{5}{|l|}{ Lymnaeidae } \\
\hline Physa sp. & 0.07 & 2.78 & 0.0278 & A \\
\hline Fossaria sp. & 0.07 & 1.39 & 0.014 & A \\
\hline \multicolumn{5}{|l|}{ Planorbidae } \\
\hline Helisoma $\mathrm{sp}$ & 0.83 & 1.39 & 0.014 & A \\
\hline Quilópodos Geophilomorpha & 0.63 & 2.78 & 0.0278 & $\mathrm{~T}$ \\
\hline \multicolumn{5}{|l|}{ Isópodos } \\
\hline Oniscidae & 0.77 & 4.17 & 0.042 & $\mathrm{~T}$ \\
\hline \multicolumn{5}{|l|}{ Arácnidos } \\
\hline Amaurobiidae & 0.02 & 1.39 & 0.014 & $\mathrm{~T}$ \\
\hline \multicolumn{5}{|l|}{ Buthidae } \\
\hline Centruroides l. limpidus & 2.82 & 4.17 & 0.042 & $\mathrm{~T}$ \\
\hline Dipluridae & 0.05 & 2.78 & 0.0278 & $\mathrm{~T}$ \\
\hline Dysderidae & 0.03 & 2.78 & 0.0278 & $\mathrm{~T}$ \\
\hline Lycosidae & 4.15 & 26.39 & 0.264 & $\mathrm{~T}$ \\
\hline Pholcidae & 0.01 & 1.39 & 0.014 & $\mathrm{~T}$ \\
\hline Salticidae & 0.02 & 1.39 & 0.014 & $\mathrm{~T}$ \\
\hline Tetragnathidae & 0.14 & 1.39 & 0.014 & $\mathrm{~T}$ \\
\hline Theridiidae & 0.05 & 4.17 & 0.042 & $\mathrm{~T}$ \\
\hline Uloboridae & 0.06 & 1.39 & 0.014 & $\mathrm{~T}$ \\
\hline Zodariidae & 0.14 & 1.39 & 0.014 & $\mathrm{~T}$ \\
\hline Indeterminados & 0.03 & 2.78 & 0.0278 & $\mathrm{~T}$ \\
\hline \multicolumn{5}{|l|}{ Opiliones } \\
\hline \multicolumn{5}{|l|}{ Sclerosomatidae } \\
\hline Leiobonum sp. & 1.82 & 13.89 & 0.139 & $\mathrm{~T}$ \\
\hline \multicolumn{5}{|l|}{ Insectos } \\
\hline Blattidae & 0.37 & 4.17 & 0.042 & $\mathrm{~T}$ \\
\hline \multicolumn{5}{|l|}{ Coleoptera } \\
\hline Cantharidae & 0.26 & 4.17 & 0.042 & $\mathrm{~T}$ \\
\hline \multicolumn{5}{|l|}{ Carabidae } \\
\hline Selenophores sp. & 0.08 & 1.39 & 0.014 & $\mathrm{~T}$ \\
\hline Stenomorphus sp. & 0.16 & 1.39 & 0.014 & $\mathrm{~T}$ \\
\hline Indeterminados & 0.93 & 11.11 & 0.111 & $\mathrm{~T}$ \\
\hline
\end{tabular}


Continúa Anexo

\begin{tabular}{|c|c|c|c|c|}
\hline Componentes alimentarios & PI (\%) & FO (\%) & IA & Hábito \\
\hline \multicolumn{5}{|l|}{ Cicindelidae } \\
\hline Cicindella sp. & 1.05 & 8.33 & 0.083 & $\mathrm{~T}$ \\
\hline \multicolumn{5}{|l|}{ Curculionidae } \\
\hline Auleutes sp. & 0.05 & 1.39 & 0.014 & A \\
\hline Rhynchophorinae & 0.13 & 2.78 & 0.028 & $\mathrm{~T}$ \\
\hline \multicolumn{5}{|l|}{ Dryophthoridae } \\
\hline Rhodobaenus sp. & 0.06 & 1.39 & 0.014 & $\mathrm{~T}$ \\
\hline Elateridae & 0.29 & 5.56 & 0.056 & $\mathrm{~T}$ \\
\hline \multicolumn{5}{|l|}{ Hydrophilidae } \\
\hline Hydrophilus sp. (larvas) & 1.32 & 12.50 & 0.125 & $\mathrm{~A}$ \\
\hline Lampiridae & 0.07 & 2.78 & 0.028 & $\mathrm{~T}$ \\
\hline \multicolumn{5}{|l|}{ Melolonthidae } \\
\hline Cyclocephala sp. & 0.74 & 2.78 & 0.028 & $\mathrm{~T}$ \\
\hline Diplotaxis sp. & 0.06 & 1.39 & 0.014 & $\mathrm{~T}$ \\
\hline \multicolumn{5}{|l|}{ Scarabeidae } \\
\hline Aphodius sp. & 0.02 & 2.78 & 0.028 & $\mathrm{~T}$ \\
\hline Staphilinidae & 0.02 & 2.78 & 0.028 & $\mathrm{~T}$ \\
\hline Tenebrionidae & 0.19 & 2.78 & 0.028 & $\mathrm{~T}$ \\
\hline Indeterminados & 0.12 & 8.33 & 0.083 & $\mathfrak{x}$ \\
\hline \multicolumn{5}{|l|}{ Colémbolos } \\
\hline \multicolumn{5}{|l|}{ Sminthurididae } \\
\hline Sminthurodes sp. & 0.01 & 2.78 & 0.028 & A \\
\hline \multicolumn{5}{|l|}{ Dermápteros } \\
\hline \multicolumn{5}{|l|}{ Forficulidae } \\
\hline Doru lineare & 1.02 & 8.33 & 0.083 & $\mathrm{~T}$ \\
\hline \multicolumn{5}{|l|}{ Dípteros } \\
\hline \multicolumn{5}{|l|}{ Ceratopogonidae } \\
\hline Dasyhelea saltensis & 0.53 & 2.78 & 0.028 & $\mathrm{~T}$ \\
\hline Chironomidae (larvas) & 0.21 & 2.78 & 0.028 & A \\
\hline \multicolumn{5}{|l|}{ Culicidae } \\
\hline Culex sp. & 0.14 & 1.39 & 0.014 & $\mathrm{~T}$ \\
\hline \multicolumn{5}{|l|}{ Dolichopodidae } \\
\hline Dolichopus sp. & 0.02 & 2.78 & 0.028 & $\mathrm{~T}$ \\
\hline \multicolumn{5}{|l|}{ Drosophilidae } \\
\hline Drosophila sp. & 0.04 & 2.78 & 0.028 & $\mathrm{~T}$ \\
\hline \multicolumn{5}{|l|}{ Lycoridae } \\
\hline Sciara sp. & 0.09 & 5.56 & 0.056 & $\mathrm{~T}$ \\
\hline \multicolumn{5}{|l|}{ Simuliidae } \\
\hline Simulium sp. & 6.73 & 13.89 & 0.139 & A \\
\hline \multicolumn{5}{|l|}{ Stratiomyidae } \\
\hline Stratiomys sp. & 0.16 & 2.78 & 0.028 & $\mathrm{~T}$ \\
\hline \multicolumn{5}{|l|}{ Tipulidae } \\
\hline Tipula sp. & 0.43 & 8.33 & 0.083 & A \\
\hline
\end{tabular}


Continúa Anexo

\begin{tabular}{|c|c|c|c|c|}
\hline Componentes alimentarios & PI (\%) & FO (\%) & IA & Hábito \\
\hline \multicolumn{5}{|l|}{ Efemerópteros } \\
\hline \multicolumn{5}{|l|}{ Baetidae } \\
\hline Baetodes sp. (larvas) & 0.02 & 2.78 & 0.028 & A \\
\hline Camelobaetidius sp. (larvas) & 0.02 & 1.39 & 0.014 & A \\
\hline \multicolumn{5}{|l|}{ Leptohyphidae } \\
\hline Leptohyphes sp. (larvas) & 0.01 & 1.39 & 0.014 & A \\
\hline Tricorythodes sp. (larvas) & 0.01 & 1.39 & 0.014 & A \\
\hline \multicolumn{5}{|l|}{ Hemípteros } \\
\hline \multicolumn{5}{|l|}{ Aetalionidae } \\
\hline Aetalion sp. & 0.16 & 6.94 & 0.069 & $\mathrm{~T}$ \\
\hline \multicolumn{5}{|l|}{ Anthocoridae } \\
\hline Anthocoris sp. & 0.02 & 1.39 & 0.014 & $\mathrm{~T}$ \\
\hline Coreidae & 0.91 & 4.17 & 0.042 & $\mathrm{~T}$ \\
\hline Corixidae & 0.49 & 1.39 & 0.014 & $\mathrm{~T}$ \\
\hline \multicolumn{5}{|l|}{ Gelastocoridae } \\
\hline Nerthra sp. & 1.07 & 5.56 & 0.056 & A \\
\hline Lygaeidae & 1.31 & 16.67 & 0.167 & $\mathrm{~T}$ \\
\hline Miridae & 0.01 & 1.39 & 0.014 & $\mathrm{~T}$ \\
\hline Nabidae & 0.02 & 1.39 & 0.014 & $\mathrm{~T}$ \\
\hline Ranatra sp. & 0.20 & 2.78 & 0.028 & A \\
\hline Pentatomidae & 0.04 & 1.39 & 0.014 & $\mathrm{~T}$ \\
\hline Reduviidae & 0.13 & 4.17 & 0.042 & $\mathrm{~T}$ \\
\hline \multicolumn{5}{|l|}{ Veliidae } \\
\hline Microvelia sp. & 0.02 & 1.39 & 0.014 & A \\
\hline Rhagovellia sp. & 0.01 & 1.39 & 0.014 & $\mathrm{~T}$ \\
\hline Indeterminados & 0.57 & 5.56 & 0.056 & $æ$ \\
\hline \multicolumn{5}{|l|}{ Himenópteros } \\
\hline Adrenidae & 0.07 & 2.78 & 0.028 & $\mathrm{~T}$ \\
\hline Chalcididae & 0.04 & 1.39 & 0.014 & $\mathrm{~T}$ \\
\hline Figitidae & 0.03 & 1.39 & 0.014 & $\mathrm{~T}$ \\
\hline \multicolumn{5}{|l|}{ Formicidae } \\
\hline Atta mexicana & 0.11 & 2.78 & 0.028 & $\mathrm{~T}$ \\
\hline Camponotus atriceps & 0.47 & 8.33 & 0.083 & $\mathrm{~T}$ \\
\hline Nomamyrmex esenbecki & 0.05 & 4.17 & 0.042 & $\mathrm{~T}$ \\
\hline Pheidole sp. & 0.02 & 1.39 & 0.014 & $\mathrm{~T}$ \\
\hline Solenopsis gemminata & 0.20 & 5.56 & 0.056 & $\mathrm{~T}$ \\
\hline Sphecidae & 0.14 & 1.39 & 0.014 & $\mathrm{~T}$ \\
\hline Vespidae & 0.64 & 4.17 & 0.042 & $\mathrm{~T}$ \\
\hline Indeterminados & 0.003 & 1.39 & 0.014 & $\mathrm{~T}$ \\
\hline \multicolumn{5}{|l|}{ Homópteros } \\
\hline Cicadellidae & 1.16 & 29.17 & 0.292 & $\mathrm{~T}$ \\
\hline Penthiminae & 0.22 & 1.39 & 0.014 & $\mathrm{~T}$ \\
\hline Typhlocybinae & 0.07 & 1.39 & 0.014 & $\mathrm{~T}$ \\
\hline
\end{tabular}


Concluye Anexo

\begin{tabular}{|c|c|c|c|c|}
\hline Componentes alimentarios & PI (\%) & FO (\%) & IA & Hábito \\
\hline Cicadidae & 1.31 & 8.33 & 0.083 & $\mathrm{~T}$ \\
\hline Delphacidae & 0.03 & 2.78 & 0.028 & $\mathrm{~T}$ \\
\hline \multicolumn{5}{|l|}{ Membracidae } \\
\hline Bilimekia sp. & 0.77 & 4.17 & 0.042 & $\mathrm{~T}$ \\
\hline \multicolumn{5}{|l|}{ Lepidópteros } \\
\hline Noctuidae & 16.60 & 52.77 & 0.528 & $\mathrm{~T}$ \\
\hline Indeterminados & 8.53 & 26.39 & 0.264 & $æ$ \\
\hline \multicolumn{5}{|l|}{ Megalopteros } \\
\hline \multicolumn{5}{|l|}{ Corydalidae } \\
\hline Corydalus texanus & 19.54 & 16.67 & 0.167 & ж \\
\hline \multicolumn{5}{|l|}{ Odonatos } \\
\hline Aehsnidae & 1.02 & 2.78 & 0.028 & Ж \\
\hline \multicolumn{5}{|l|}{ Coenagrionidae } \\
\hline Argia sp. (larvas) & 0.26 & 2.78 & 0.028 & A \\
\hline \multicolumn{5}{|l|}{ Gomphidae } \\
\hline Dromogompus sp. (larvas) & 0.66 & 1.39 & 0.014 & A \\
\hline Libellulidae & 1.42 & 8.33 & 0.083 & ж \\
\hline Indeterminados & 1.76 & 2.78 & 0.028 & ж \\
\hline \multicolumn{5}{|l|}{ Ortópteros } \\
\hline Acrididae & 0.07 & 1.39 & 0.014 & $\mathrm{~T}$ \\
\hline Gryllidae & 1.28 & 11.11 & 0.111 & $\mathrm{~T}$ \\
\hline Indeterminados & 0.52 & 4.17 & 0.042 & $\mathrm{~T}$ \\
\hline \multicolumn{5}{|l|}{ Tricópteros } \\
\hline \multicolumn{5}{|l|}{ Hydropsichidae } \\
\hline Hydropsyche sp. (larvas) & 0.01 & 1.39 & 0.014 & A \\
\hline Leptonema sp. & 0.01 & 2.78 & 0.028 & $\mathrm{~T}$ \\
\hline Hydroptilidae & 0.08 & 1.39 & 0.014 & $\mathrm{~T}$ \\
\hline \multicolumn{5}{|l|}{ Odontoceridae } \\
\hline Marilia sp. & 0.09 & 1.39 & 0.014 & $\mathrm{~T}$ \\
\hline \multicolumn{5}{|l|}{ Peces } \\
\hline \multicolumn{5}{|l|}{ Cichlidae } \\
\hline Cichlasoma nigrofasciatum & 0.18 & 1.39 & 0.014 & A \\
\hline Indeterminados & 0.06 & 1.39 & 0.014 & A \\
\hline \multicolumn{5}{|l|}{ Anfibios anuros } \\
\hline \multicolumn{5}{|l|}{ Ranidae } \\
\hline Lithobates sp. (larvas) & 4.79 & 5.56 & 0.056 & A \\
\hline Materia animal mixta indeterminada & 2.86 & 26.39 & 0.263 & $æ$ \\
\hline Materia mineral (piedras) & - & 6.94 & - & \\
\hline
\end{tabular}

Recibido: 12 de diciembre de 2006

Aceptado: 15 de octubre de 2007 